\title{
Kinematics of the southern galaxy cluster Abell 3733*
}

\author{
J.M. Solanes ${ }^{1,2}$ and P. Stein ${ }^{1}$ \\ 1 Departament d'Astronomia i Meteorologia, Universitat de Barcelona, Av. Diagonal 647, E-08028 Barcelona, Spain \\ 2 Departament d'Enginyeria Informàtica, Universitat Rovira i Virgili, Carretera de Salou S/M, E-43006 Tarragona, Spain \\ e-mail: solanes@pcess1.am.ub.es, paul@pcess2.am.ub.es
}

Received January 16; accepted March 2, 1998

\begin{abstract}
We report radial velocities for 99 galaxies with projected positions within $30^{\prime}$ of the center of the cluster A3733 obtained with the MEFOS multifiber spectrograph at the 3.6-m ESO telescope. These measurements are combined with 39 redshifts previously published by Stein (1996) to build a collection of 112 galaxy redshifts in the field of A3733, which is used to examine the kinematics and structure of this cluster. We assign cluster membership to 74 galaxies with heliocentric velocities in the interval $10500-13000 \mathrm{~km} \mathrm{~s}^{-1}$. From this sample of cluster members, we infer a heliocentric systemic velocity for A3733 of $11653_{-76}^{+74} \mathrm{~km} \mathrm{~s}^{-1}$, which implies a mean cosmological redshift of 0.0380 , and a velocity dispersion of $614_{-30}^{+42} \mathrm{~km} \mathrm{~s}^{-1}$. The application of statistical substructure tests to a magnitude-limited subset of the latter sample reveals evidence of non-Gaussianity in the distribution of ordered velocities in the form of lighter tails and possible multimodality. Spatial substructure tests do not find, however, any significant clumpiness in the plane of the sky, although the existence of subclustering along the line-ofsight cannot be excluded.
\end{abstract}

Key words: galaxies: clusters: individual: A 3733 galaxies: distances and redshifts

\section{Introduction}

The rapid development of multifiber spectroscopy in recent years has made possible the simultaneous acquisition of large numbers of galaxy spectra. The obtention of extensive and complete redshift data bases for clusters

\footnotetext{
Send offprint requests to: J.M. Solanes

* Based on observations made at the European Southern Observatory, La Silla, Chile.

1 Table 1 is only available in electronic form at the CDS via anonymous ftp to cdsarc.u-strasbg.fr (130.79.128.5) or via http://cdsweb.u-strasbg.fr/Abstract.html
}

of galaxies has hastened the investigation of the physical properties of their visual component which, in turn, is allowing for a better understanding of the characteristics of the dark matter distribution on Mpc scales. Here, we report a total of 104 redshift measurements for 99 galaxies in the field of A3733 and use these data, in combination with a previously published sample of 39 redshifts, to perform a kinematic and spatial analysis of the central regions of this cluster.

A3733 is a southern galaxy cluster listed in the ACO catalog (Abell et al. 1989) as of intermediate Abell's morphological type and richness class $R=1$. This cluster hosts a central cD galaxy, included in the Wall \& Peacock (1985) all-sky catalog of brightest extragalactic radio sources at $2.7 \mathrm{GHz}$, which has led to its classification as of Bautz-Morgan type I-II (Bautz \& Morgan 1970). A3733 is also one of the 107 nearby rich ACO clusters $(R \geq 1, z \leq 0.1)$ included by Katgert et al. (1996) in the ESO Nearby Cluster Survey (ENACS), as well as one of the X-ray-brightest Abell clusters detected in the ROSAT All-Sky Survey by Ebeling et al. (1996).

The only major kinematical study of A3733 done so far is that of Stein (1997). From a sample of 27 cluster members located within $r \lesssim 16^{\prime}$ from the cluster center, this author has found no evidence of significant substructure in the cluster core. This study of A3733, which is part of a more general investigation of the frequency of substructure in the cluster cores from an optical spectroscopic survey conducted on a sample of 15 nearby $(0.01 \lesssim z \lesssim 0.05)$ galaxy clusters (Stein 1996), is based on a dataset that has many characteristics in common with the ENACS data gathered for the same field. Indeed, the two datasets have been obtained with the OPTOPUS multifiber spectrograph at the ESO 3.6-m telescope and cover essentially the same area on the sky. Besides, they have also a very similar number of galaxies: 39 and 44, respectively (28 of which are shared).

The MEFOS redshift dataset for A3733 reported in this paper contains two and a half times the number of 
galaxy radial velocities reported by Stein (1996), including 26 reobservations, while it covers a circular region around the center of A3733 four times larger. Furthermore, its high degree of completeness offers the possibility of extracting a complete magnitude-limited subset with a number of galaxies large enough for its use on statistical analysis. The plan of the paper is as follows. In Sect. 2 we discuss the MEFOS spectroscopic observations and data reduction, and present a final sample with 112 entries built by the combination of the MEFOS and Stein's (1996) data. Section 3 begins with a brief description of the tools which will be used for the analysis of the data. Next, we identify the galaxies in our sample that belong to A3733, and use this dataset and a nearly complete magnitude-limited subset of it to examine the kinematical properties and structure of the central regions of the cluster. Section 4 summarizes the results of our study.

\section{MEFOS observations and data reduction}

A total of 104 redshift measurements for 99 galaxies within a circular region of $30^{\prime}$ around the radio position of the cluster cD, RA $=20^{\mathrm{h}} 58^{\mathrm{m}} 39^{\mathrm{s}} .0$ and $\mathrm{Dec}=-28^{\circ} 15^{\prime} 22^{\prime \prime}$ (Tadhunter et al. 1993), were obtained using the MEFOS multifiber spectrograph at the 3.6-m ESO telescope at La Silla (Chile). The observations were carried out on May 23-26, 1995 during an observing run whose main target was the dwarf galaxy population of the Centaurus cluster (see Stein et al. 1997). The MEFOS instrument has a circular field of view of $1^{\circ}$ and 29 fiber arms which carry two spectral fibers of 2.5 aperture for simultaneous object and sky acquisition, and one image fiber of $36^{\prime \prime} \times 36^{\prime \prime}$ for the interactive repositioning of the spectral fibers. A grating with 300 lines $\mathrm{mm}^{-1}$ was used to produce spectra in the range between 3800 and $6100 \AA$ with a typical resolution of ca. $10 \AA$. The detector was a TI $512 \times 512$ CCD chip.

The raw CCD spectra were reduced using the MIDAS package through several steps which include cleaning from defects, cosmic ray removal, flat-fielding, one-dimensional extraction, and wavelength calibration using a He-Ne lamp before and after each exposure. The sky subtraction was performed subtracting from each one of the object spectra the mean of the output of all the fibers positioned on blank sky positions from the corresponding exposure. Prior to sky subtraction the signal of each spectrum (including the sky spectra) was scaled with respect to the intrinsic transmission efficiency of the corresponding fiber, which had been determined using the average over the observed fields of the signal under the O I emission line at $5577.4 \AA$.

After the final one-dimensional spectra had been extracted, velocities were computed either from emission lines or from absorption lines, or from both. Emission-line redshifts were obtained from galaxies with at least two clearly visible emission lines (mostly $\mathrm{O}$ II, $\mathrm{H} \beta$, and $\mathrm{O}$ III).
Their redshifted positions were determined from fits with a Gaussian superposed onto a quadratic polynomial approximating the local continuum. The final redshift of a galaxy was then computed as the unweighted mean over the $n$ emission lines present in its spectrum. Since the errors in the redshift measurement of each single line are essentially dominated by uncertainties in the wavelength calibration (Stein 1996), individual measurement errors were taken equal to $100 \mathrm{~km} \mathrm{~s}^{-1}$, independently of line strength. Accordingly, an uncertainty of $100 / \sqrt{n} \mathrm{~km} \mathrm{~s}^{-1}$ was assigned to emission-line redshifts. Absorption-line redshifts were obtained using the standard cross-correlation algorithm described by Tonry \& Davis (1979). This technique requires the previous removal of both galaxy emission lines and strong night-sky lines, and the transformation of the spectral continuum to a constant level of zero. Special care was taken that the continuum subtraction did not create spurious features of low spatial frequency which could be confused with broad, superposed absorption lines. For the determination of the zero-point shift one single template was constructed by merging 20 galaxy spectra with high $\mathrm{S} / \mathrm{N}$ and well known redshifts. Only normalized cross-correlation peaks of height 0.25 or larger were considered as significant. Both emission-line and cross-correlation redshifts were then corrected to heliocentric values.

The 26 galaxies observed also by Stein (1996) in the field of A3733 with the OPTOPUS spectrograph were used to determine the scaling factor of the internal errors estimated in the cross-correlation procedure, resulting in external errors of typically $40-50 \mathrm{~km} \mathrm{~s}^{-1}$. These same galaxies gave a mean velocity difference of $-23 \mathrm{~km} \mathrm{~s}^{-1}$ (the MEFOS redshifts being typically smaller), consistent with zero to within the reported measurement errors. Only for 5 of our galaxies we could measure both emission-line and cross-correlation redshifts. Again, an excellent consistency was found between the two kinds of measurements.

The cross-correlation and emission-line radial velocities for the 99 galaxies observed with the MEFOS spectrograph in the field of A3733 are listed in Cols. (4) and (5) of Table 1, together with their estimated external errors. Columns (6) and (7) give the same information for the 39 galaxies observed with the OPTOPUS instrument by Stein (1996). Column (8) lists the final radial velocities and their estimated uncertainties which result form a weighted average of the data in Cols. (4)-(7). The combination of these two samples gives a total of 112 entries, which will be used in the following section to examine the kinematical properties and structure of A3733. This is about three times the number of galaxies used in the previous study by Stein (1997). The first three columns of Table 1 contain the celestial coordinates for the epoch B1950.0 and the $b_{\mathrm{J}}$ magnitudes from the COSMOS catalog kindly provided by H. MacGillivray. The completeness in apparent magnitude of the final dataset is high, with percentages of $100,92,75,63$, and $50 \%$ of all known 
(COSMOS) galaxies in the same region of the sky covered by our observations at the $b_{\mathrm{J}}$ magnitude limits of 17.0 , $17.5,18.0,18.5$, and 19.0, respectively.

\section{Kinematic and spatial analysis}

Following the recommendations of Beers et al. (1990), we will characterize the velocity distribution of our cluster sample by means of the biweight estimators of central location (i.e., systemic velocity), $\bar{V}$, and scale (i.e., velocity dispersion), $\sigma$. We will assign errors to these estimates equal to the $68 \%$ bias-corrected bootstrap confidence intervals inferred from 10000 resamplings. The program ROSTAT, kindly provided by $\mathrm{T}$. Beers, will be used for all these calculations.

The ROSTAT program includes also a wide variety of statistical tests, which can be used to assess the consistency of the empirical line-of-sight velocity distribution of the A3733 members (see next subsection) with draws from a single Gaussian parent population. A fair representation of the overall results of the ROSTAT tests will be given by quoting the value of the statistic and associated probability for the canonical $B_{1}$ and $B_{2}$ tests, which measure, respectively, the skewness (asymmetry) and curtosis (elongation) of the velocity distribution, and for the AndersonDarling $A^{2}$ omnibus test. Definitions of these tests can be found in Yahil \& Vidal (1977) and D'Agostino (1986). The Gaussianity tests will be complemented by the Dip test of Hartigan \& Hartigan (1985), which tests the hypothesis that a sample is drawn from a unimodal (though not necessarily Gaussian) parent distribution, and by the search of individual weighted gaps, $g_{*}$, in the velocity distribution of size 2.75 or larger (for a definition of weighted gap see, for instance, Beers et al. 1990). Individual weighted gaps this large are highly significant since they arise less than $1 \%$ of the time in random draws from a Gaussian distribution, independently of sample size. We refer the reader to the listed sources and references therein for a detailed explanation of these statistical techniques.

We will investigate also the presence of substructure in the spatial distribution of galaxies by means of two powerful tests. First, we will apply a 2D test developed by Salvador-Solé et al. (1993), hereafter referred to as the SSG test, which relies exclusively on the projected positions of galaxies on the sky (though velocity information is required to define strict cluster membership). This test produces two different estimates of the projected number density profile of the cluster, $N_{\mathrm{dec}}(r)$ and $N_{\mathrm{dir}}(r)$, which are, respectively, sensitive and insensitive to the existence of correlation in the galaxy positions relative to the cluster background density. The subscript "dec" identifies the density profile obtained via the deconvolution of the histogram of intergalaxy separations, while the subscript "dir" applies to the density profile arising directly from the integral of the histogram of clustercentric distances of the cluster galaxies (Eqs. (4) and (6), respectively, in Salvador-Solé et al. 1993). The two profiles are convolved with a window of smoothing size $\lambda_{\min }$ corresponding to the minimum resolution-length imposed by the calculation of $N_{\text {dec }}(r)$. The significance of substructure is estimated from the null hypothesis that $N_{\mathrm{dec}}(r)$ arises from a Poissonian realization of some (unknown) theoretical density profile which has led to the observed radial distribution of galaxies. The probability of this being the case is calculated by means of the statistic:

$\chi^{2}=\frac{\left(N_{\mathrm{dec}}(0)-N_{\mathrm{dir}}(0)\right)^{2}}{2 S^{2}(0)}$

for one degree of freedom. In Eq. (1), $N_{\mathrm{dec}}(0)$ and $N_{\mathrm{dir}}(0)$ are the central values of the respective density profiles of the cluster, while $S^{2}(0)$ is the central value of the radial run of the variance of $N_{\text {dir }}(r)$ calculated from a set of simulated clusters convolved to the $\lambda_{\min }$ imposed by $N_{\mathrm{dec}}(r)$. The simulated clusters are generated by the azimuthal scrambling of the observed galaxy positions around the center of the cluster, i.e., by randomly shuffling between 0 and $2 \pi$ the azimuthal angle of each galaxy, while maintaining its clustercentric distance unchanged. It must be stressed, however, that the sensitivity of the SSG test is not affected by deviations of the spatial distribution of the galaxy sample under scrutiny from circular symmetry (see Salvador-Solé et al. 1993). It is also worth noting that this test does not require a priori assumptions on the form of the true projected number density profile of the cluster, nor on the number and size of the subgroups that might be present in the data.

The second spatial substructure test that will be applied to our data is the 3D Dressler \& Shectman (1988b; DS) test, which is sensitive to local kinematic deviations in the projected galaxy spatial distribution. The DS test assigns a local estimate of the velocity mean, $\bar{V}_{\text {loc }}$, and dispersion, $\sigma_{\text {loc }}$, to each galaxy with a measured radial velocity. These values are then compared with the values of the kinematical parameters for the entire sample. The statistic used to quantify the presence of substructure is the sum of the local kinematic deviations for each galaxy, $\delta_{i}$, over the $N$ cluster members, which we will calculate through the expression:

$$
\begin{aligned}
\Delta & =\sum_{i=1}^{N} \delta_{i} \\
& =\sum_{i=1}^{N}\left[\frac{N_{\mathrm{kern}}+1}{\sigma^{2}}\left(\left(\bar{V}_{\mathrm{loc}, i}-\bar{V}\right)^{2}+\left(\sigma_{\mathrm{loc}, i}-\sigma\right)^{2}\right)\right]^{1 / 2}
\end{aligned}
$$

where $N_{\text {kern }}=\operatorname{nint}(\sqrt{N}), \operatorname{nint}(x)$ stands for the integer nearest to $x$, maximizes the sensitivity of the DS test to significant substructure (Bird 1994). To avoid the formulation of any hypothesis on the form of the velocity distribution of the parent population, the DS test calibrates the $\Delta$ statistic by means of Monte-Carlo simulations that 

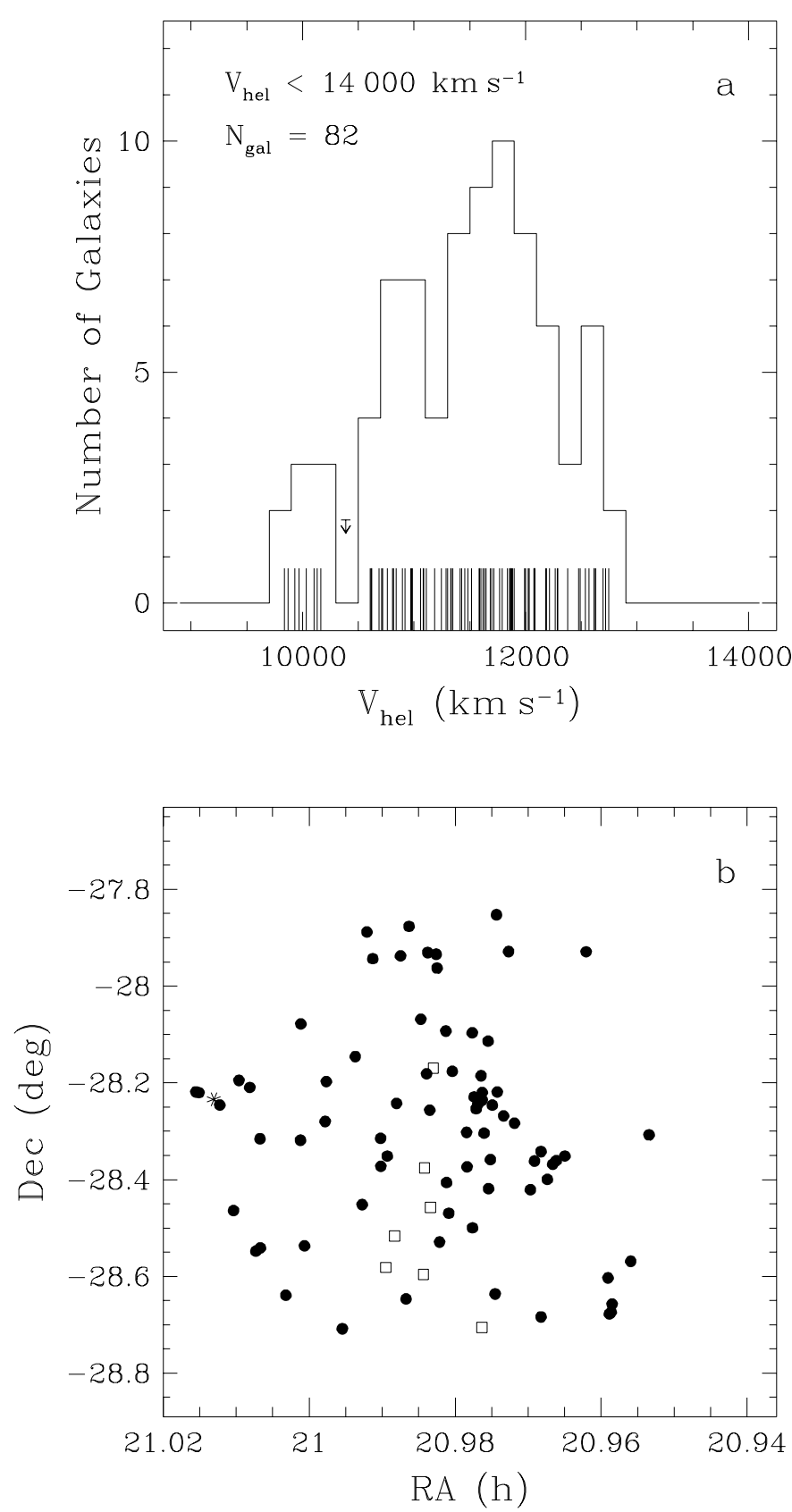

Fig. 1. a) Stripe density plot and velocity histogram of the galaxies with $V_{\text {hel }}<14000 \mathrm{~km} \mathrm{~s}^{-1}$ in the A3733 sample. The arrow marks the location of a highly significant weighted gap $(p=0.001)$ in the velocity distribution. b) Corresponding spatial distribution. The 7 members of a suspect foreground group are identified by open squares, while the asterisk marks the galaxy with the lowest $V_{\text {hel }}$. Filled circles identify our choice of strict cluster members randomly shuffle the velocities of the galaxies while keeping their observed positions fixed. In this way any existing correlation between velocities and positions is destroyed. The probability of the null hypothesis that there are no local correlations between the position and velocity of the cluster members is given in terms of the fraction of simulated clusters for which their cumulative deviation, $\Delta_{\text {sim }}$, is smaller than the observed value, $\Delta_{\text {obs }}$. Again, we refer the reader to the quoted references for further details on the two spatial substructure tests used in the present analysis.

\subsection{The sample of cluster members}

Before we can investigate the presence of substructure in A3733 we need to assign cluster membership to the galaxies in our sample. Examination of the radial velocities of the 112 galaxies listed in Table 1 allows the exclusion of 30 obvious interlopers (all background galaxies and groups), which are separated by more than $6500 \mathrm{~km} \mathrm{~s}^{-1}$ from the main velocity group. Subsequent membership assignment for the remaining 82 galaxies is based on the their velocity distribution and projected positions, displayed in Figs. 1a and 1b, respectively. These figures show the existence of 8 objects with velocities smaller than $10500 \mathrm{~km} \mathrm{~s}^{-1}$ separated from the other galaxies by a gap in heliocentric velocity of $\sim 450 \mathrm{~km} \mathrm{~s}^{-1}$. Seven of these galaxies appear also to be concentrated on a small area of the sky. The cluster diagnostics described at the beginning of this section reveal that the above gap in velocity corresponds to an individually large normalized gap of size 3.39 in the 82 ordered velocities. The "per-gap" probability for a weighted gap this size is only 0.001 . This and the fact that the suspected foreground group of 7 galaxies has a velocity dispersion of only $73 \mathrm{~km} \mathrm{~s}^{-1}$ suggest that it might constitute a separate dynamical entity. Accordingly, we chose to consider bona fide A3733 members the 74 galaxies in our sample with heliocentric velocities between 10500 and $13000 \mathrm{~km} \mathrm{~s}^{-1}$. Note that we are excluding also from cluster membership the remaining foreground object with the lowest measured radial velocity. From the set of cluster members, we obtain $\bar{V}_{\text {hel }}=11653_{-76}^{+74} \mathrm{~km} \mathrm{~s}^{-1}$ and $\sigma=614_{-30}^{+42} \mathrm{~km} \mathrm{~s}^{-1}$ after applying relativistic and measurement error corrections (Danese et al. 1980). These values are compatible, within the adopted uncertainties, with the values $\bar{V}_{\text {hel }}=11716 \pm 103 \mathrm{~km} \mathrm{~s}^{-1}$ and $\sigma=$ $522 \pm 84 \mathrm{~km} \mathrm{~s}^{-1}$ obtained in the previous analysis of this cluster by Stein (1997) from a sample containing 27 of the current cluster members. The mean heliocentric velocity calculated for A3733 results in a mean cosmological redshift of $\bar{z}_{\mathrm{CMB}}=0.0380$ after correction to the CMB rest frame (Kogut et al. 1993). At the cosmological distance of A3733, one Abell radius, $r_{\mathrm{A}}\left(\equiv 1.5 h^{-1} \mathrm{Mpc}\right)$, is equal to 0.805 degrees. The subset of 82 galaxies with $V_{\text {hel }}<13000 \mathrm{kms}^{-1}$ has $\bar{V}_{\text {hel }}=11532_{-89}^{+94} \mathrm{~km} \mathrm{~s}^{-1}$, 
$\sigma=754_{-48}^{+65} \mathrm{~km} \mathrm{~s}^{-1}, \bar{z}_{\mathrm{CMB}}=0.0385$, and $r_{\mathrm{A}}=0.812$ degrees.

The values of the kinematical parameters of the cluster have been calculated without taking into account its dynamical state. Indeed, the visual inspection of Fig. 1a yields suggestive indication of deviation of the velocity distribution from Gaussianity in the form of lighter tails and a hint of multimodality. The dictum of the $B_{2}$ statistic, which indicates the amount of elongation in a sample relative to the Gaussian, confirms the platycurtic behavior (i.e., $B_{2}<3$ ) of the velocity histogram giving only a 0.001 probability that it could have arisen by chance from a parent Gaussian population. Nevertheless, the results of the $B_{1}$ and $A^{2}$ tests do not indicate significant departures from normality. As for the possible multimodality, the Dip test cannot reject the unimodal hypothesis, nor we detect the presence of highly significant large gaps in the ordered velocities.

Comparable results are obtained if we remove from the sample of cluster members those galaxies with strong emission lines in their spectrum. Indeed, the spatial distribution and kinematic properties of these latter galaxies are similar to those of the galaxies for which only crosscorrelation redshifts are available. Specifically, for the 12 cluster members with emission-line redshifts we find $\bar{V}_{\text {hel }}=11416_{-218}^{+256} \mathrm{~km} \mathrm{~s}^{-1}$ and $\sigma=694_{-101}^{+164} \mathrm{~km} \mathrm{~s}^{-1}$, while the remaining 62 galaxies have $\bar{V}_{\text {hel }}=$ $11694_{-83}^{+80} \mathrm{~km} \mathrm{~s}^{-1}$ and $\sigma=594_{-37}^{+44} \mathrm{~km} \mathrm{~s}^{-1}$.

\subsection{The magnitude-limited sample}

In order to mitigate the effects of incomplete sampling which may contaminate the results of the statistical tests, especially of those relying on local spatial information, we concentrate our subsequent analysis on the subset of 37 members of A3733 with $b_{\mathrm{J}} \leq 18$, for which our original redshift sample contains $75 \%$ of the COSMOS galaxies. This magnitude limit is chosen as a compromise between defining a sample (nearly) free of sampling biases and simultaneously having a large enough number of objects for the detection of substructure not to be affected by Poissonian errors.

For this sample, the Gaussianity tests confirm essentially the results obtained for the whole set of cluster members: the $B_{2}$ test rejects the Gaussian hypothesis at the $6 \%$ significance level, while the $B_{1}$ and $A^{2}$ tests are consistent with a parent normal population. Remarkably, the results of the other two 1D tests are now substantially different: the Dip test rejects the hypothesis of unimodality at the $4 \%$ significance level, while a large gap of size roughly $230 \mathrm{~km} \mathrm{~s}^{-1}\left(g_{*}=3.14, p=0.002\right)$ appears near the middle of the distribution $\left(V_{\text {hel }} \sim 11500 \mathrm{~km} \mathrm{~s}^{-1}\right)$ of velocities.

The kinematical complexity of the inner regions of A3733 suggested by these latter results is not reflected, however, on the spatial distribution of the galaxies. The SSG test gives, for 1000 realizations of the cluster generated by the azimuthal scrambling of the galaxy positions around the location of the $\mathrm{cD}$ (see Sect. 2), a $56 \%$ probability that there is no substructure, which is nonsignificant. The resulting $\lambda_{\min }$ of $16.7\left(\equiv 0.52 h^{-1} \mathrm{Mpc}\right)$ puts an upper limit to the half-coherence length of any possible clump that may remain undetected in the central regions of A3733. This value is above the typical scale-length of $\sim 0.3 h^{-1} \mathrm{Mpc}$ of the clumps detected by Salvador-Solé et al. (1993) in the Dressler \& Shectman's (1988a) clusters. This suggests that the presence of significant substructure in the magnitude-limited sample might be hidden by the large smoothing scale imposed by the calculation of $N_{\text {dec }}(r)$. We have investigated this possibility by applying also the SSG test to the sample containing all the 74 cluster members, for which the minimum resolutionlength reduces to only 3!69 ( $\left.\equiv 0.12 h^{-1} \mathrm{Mpc}\right)$. In spite of the fact that this latter sample is biased towards the most populated regions of A3733, therefore emphasizing any possible clumpiness of the galaxy distribution on the plane of the sky, we still obtain a $14 \%$ probability for the null hypothesis.

The DS test also points to the lack of significant substructure in the magnitude-limited sample: more than $15 \%$ of the values of the statistic $\Delta_{\text {sim }}$ obtained in 1000 MonteCarlo simulations of this sample are larger than $\Delta_{\text {obs. }}$. A visual judgment of the statistical significance of the local kinematical deviations can be done by comparing the plots in Figs. 2a-d. Figure 2a shows the spatial distribution of the galaxies superposed on their adaptive kernel density contour map (see Beers 1992 and references therein for a description of the adaptive kernel technique). The primary clump in this map is centered at the position of the $\mathrm{cD}$ galaxy and is elongated along the north-south axis; a mild density enhancement can be seen at the plot coordinates $(17,-3)$. In this figure galaxies with $V_{\text {hel }} \leq 11500 \mathrm{~km} \mathrm{~s}^{-1}$ are represented by empty circles, while solid circles mark the location of those with $V_{\text {hel }}>11500 \mathrm{~km} \mathrm{~s}^{-1}$. Although there is no strong spatial segregation among the galaxies belonging to each of these two velocity subgroups, the galaxies included in the second one dominate the central density enhancement. In Fig. $2 \mathrm{~b}$ each galaxy is identified with a circle whose radius is proportional to $\exp \left(\delta_{i}\right)$, where $\delta_{i}$ is given by Eq. (2). Hence, the larger the circle, the larger the deviation from the global values (but beware of the insensitivity of the $\delta_{i}$ 's to the sign of the deviations from the mean cluster velocity). The superposition of the projected density contours shows that most of the galaxies to the north of the density peak, and to a lesser extent those closest to the center of the eastern small density enhancement, have apparently large local deviations from the global kinematics. The remaining figures show two of the 1000 Monte-Carlo models performed: Fig. 2c corresponds to the model whose $\Delta_{\text {sim }}$ is closest to the median of the simulations, while Fig. 2 d corresponds to the 

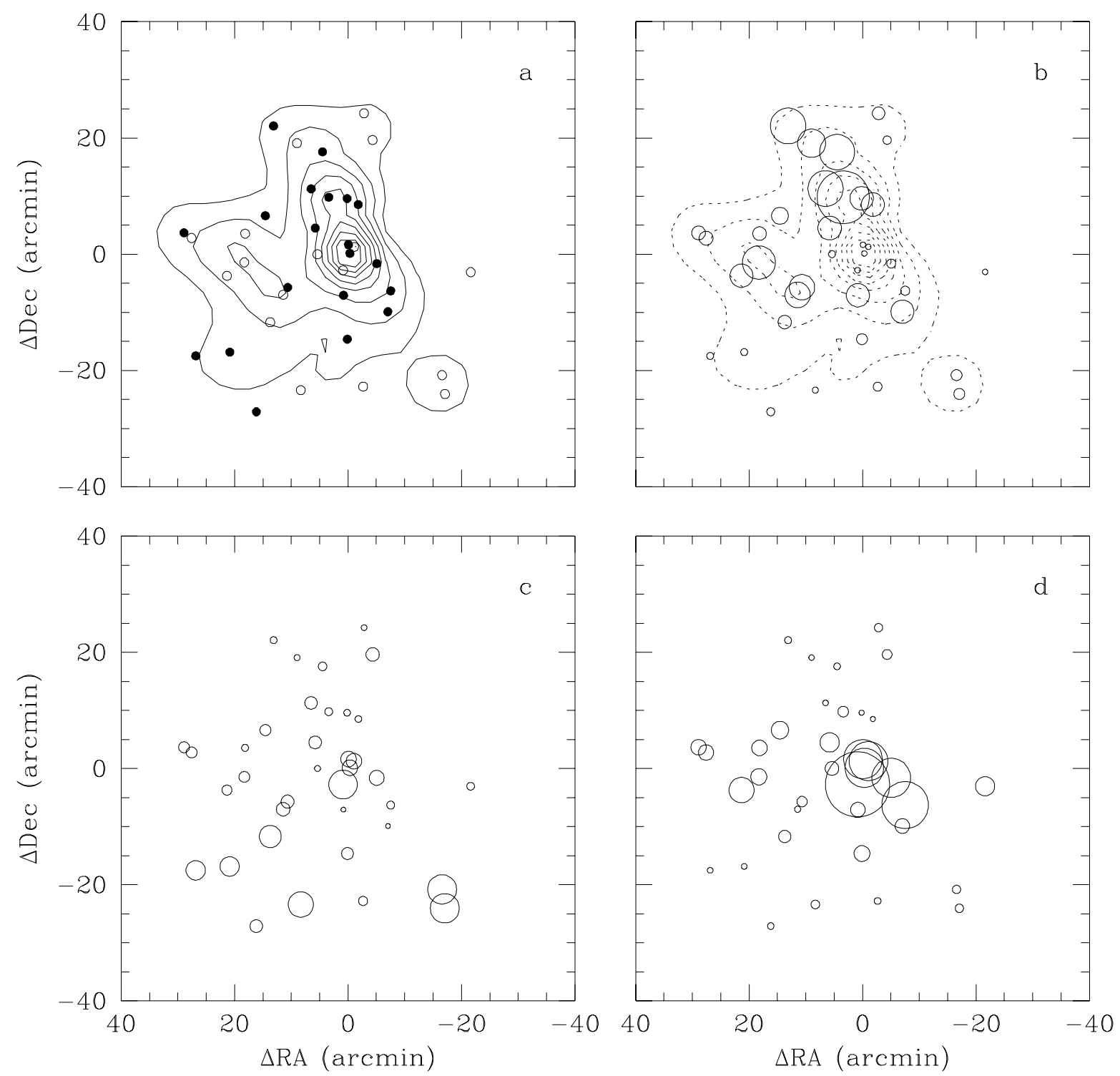

Fig. 2. a) Spatial distribution of the 37 galaxies belonging to the magnitude-limited sample $\left(b_{\mathrm{J}} \leq 18\right)$ of A3733 members. Galaxies with $V_{\text {hel }} \leq 11500 \mathrm{~km} \mathrm{~s}^{-1}$ are identified by empty circles, while solid circles mark the location of the galaxies with $V_{\text {hel }}>11500 \mathrm{~km} \mathrm{~s}^{-1}$. Curves are equally spaced contours of the adaptive kernel density contour map for this sample. The contours range from $2.1810^{-4}$ to $1.8810^{-3}$ galaxies arcmin ${ }^{-2}$. The initial smoothing scale is set to 12 '.6. b) Local deviations from the global kinematics as measured by the DS test. Open circles drawn at the position of the individual galaxies scale with the deviation of the local kinematics from the global kinematics, $\delta_{i}$, from which the test statistic $\Delta_{\mathrm{obs}}=\sum \delta_{i}$ 's is calculated (see text). The adaptive kernel contour map is superposed (dashed lines). c) and d) Monte-Carlo models of the magnitude-limited sample obtained after 1000 random shufflings of the observed velocities: c model with the cumulative deviation $\Delta_{\text {sim }}$ closest to the median of the simulations; $\mathbf{d}$ model whose $\Delta_{\text {sim }}$ is closest to the value of the upper quartile. Spatial coordinates are relative to the cluster center (see text)

Table 2. Results of the statistical tests

\begin{tabular}{cccccccccccccc}
\hline $\begin{array}{c}\text { Sample } \\
(1)\end{array}$ & $\begin{array}{c}N_{\text {gal }} \\
(2)\end{array}$ & $\begin{array}{c}B_{1} \\
(3)\end{array}$ & $\begin{array}{c}p\left(B_{1}\right) \\
(4)\end{array}$ & $\begin{array}{c}B_{2} \\
(5)\end{array}$ & $\begin{array}{c}p\left(B_{2}\right) \\
(6)\end{array}$ & $\begin{array}{c}A^{2} \\
(7)\end{array}$ & $\begin{array}{c}p\left(A^{2}\right) \\
(8)\end{array}$ & $\begin{array}{c}\text { Dip } \\
(9)\end{array}$ & $\begin{array}{c}p(\operatorname{Dip}) \\
(10)\end{array}$ & $\begin{array}{c}\chi^{2} \\
(11)\end{array}$ & $\begin{array}{c}p\left(\chi^{2}\right) \\
(12)\end{array}$ & $\begin{array}{c}\Delta_{\text {obs }} \\
(13)\end{array}$ & $\begin{array}{c}p\left(\Delta_{\text {obs }}\right) \\
(14)\end{array}$ \\
\hline Mag.-limited & 37 & 0.20 & 0.28 & 2.08 & 0.06 & 0.52 & 0.18 & 0.08 & 0.04 & 0.35 & 0.56 & 40.8 & 0.15 \\
All members & 74 & 0.00 & 0.50 & 1.99 & 0.001 & 0.51 & 0.20 & 0.03 & 0.99 & 2.19 & 0.14 & 107. & 0.003 \\
\hline
\end{tabular}


model with a $\Delta_{\text {sim }}$ closest to the value of the upper quartile. The comparison of Fig. $2 \mathrm{~b}$ with these last two figures shows that the observed local kinematical deviations are indeed not significant.

As commented in the Introduction, Stein (1997) has not found either any evidence of significant clumpiness on his A3733 OPTOPUS data (see his Table 3). Nevertheless, we caution that this previous study is restricted to the innermost $\left(r \leq 16^{\prime}\right)$ regions of the cluster and that it uses, due to the small size of the sample, all the redshifts available without regard to their completeness.

The results of all the statistical tests applied to our magnitude-limited sample are summarized in Table 2, together with the results obtained from the whole sample of cluster members, for comparison. In Col. (1) we list the name of the sample and in Col. (2) the number of galaxies in it. Columns (3)-(14) give the values of the test statistic and associated significance levels for the $B_{1}, B_{2}, A^{2}$, Dip, SSG, and DS tests, respectively. The significance levels refer to the probability that the empirical value of a given statistic could have arisen by chance from the null hypothesis. Thus, the smaller the quoted probability the more significant is the departure from it.

\section{Summary}

We have reported 104 radial velocity measurements performed with the MEFOS multifiber spectrograph at the 3.6-m ESO telescope for 99 galaxies in a region of $30^{\prime}$ around the center of the cluster A3733. To augment this data, we have combined the MEFOS measurements with 39 redshifts measured by Stein (1996) with the OPTOPUS instrument at the same telescope. This has given a final dataset with a total of 112 entries in the field of A3733. Radial velocities have been then supplemented by COSMOS $b_{\mathrm{J}}$ magnitudes and accurate sky positions in order to investigate the kinematics and structure of the central regions of the cluster.

From a sample containing 74 strict cluster members, we have derived a heliocentric systemic velocity for A3733 of $11653_{-76}^{+74} \mathrm{~km} \mathrm{~s}^{-1}$, resulting in a $\bar{z}_{\mathrm{CMB}}$ of 0.0380 , and a velocity dispersion of $614_{-30}^{+42} \mathrm{~km} \mathrm{~s}^{-1}$, in good agreement with the estimates by Stein (1997) from the OPTOPUS data alone. Statistical tests relying exclusively on the distribution of observed velocities have yield suggestive indication of the possible kinematical complexity of A3733, especially when applied to a nearly complete magnitudelimited $\left(b_{\mathrm{J}} \leq 18\right)$ sample of cluster members. Despite this result, two powerful substructure tests that incorporate spatial information have failed to detect in this latter sample any statistically significant evidence of clumpiness in the galaxy component, in agreement with the findings of a previous study based on a spatially less extended and less complete dataset. Given that the sensitivity of the spatial substructure tests we have used is reduced when the subunits are seen with small projected separations, the results of the present study cannot exclude, however, the possibility that the signs of kinematical complexity detected in the velocity histogram of A3733 might be due to the existence of galaxy subcondensations superposed along the line-of-sight.

Acknowledgements. This work has been supported by the Dirección General de Investigación Científica y Técnica, under contract PB96-0173. P.S. acknowledges partial support from a research network grant by the Commission of the European Communities.

\section{References}

Abell G.O., Corwin H.G.Jr., Olowin R.P., 1989, ApJS 70, 1

Bautz L.P., Morgan W.W., 1970, ApJ 162, L149

Beers T.C., 1992, in: Statistical Challenges in Modern Astronomy, Feigelson E.D. \& Babu G.J. (ed.). New York: Springer-Verlag, p. 111

Beers T.C., Flynn K., Gebhardt K., 1990, AJ 100, 32

Bird C.M., 1994, AJ 107, 1637

D'Agostino R.B., 1986, in: Goodness of Fit Techniques, D'Agostino R.B. \& Stephens M.A. (ed.). New York: Dekker, p. 367

Danese L., De Zotti G., di Tullio G., 1980, A\&A 82, 322

Dressler A., Shectman S.A., 1988a, AJ 95, 284

Dressler A., Shectman S.A., 1988b, AJ 95, 985 (DS)

Ebeling H., Voges W., Böhringer H., Edge A.C., Huchra J.P., Briel U.G., 1996, MNRAS 281, 799

Hartigan J.A., Hartigan P.M., 1985, Ann. Stat. 13, 70

Katgert P., et al., 1996, A\&A 310, 8

Kogut A., et al., 1993, ApJ 419, 1

Salvador-Solé E., Sanromá M., González-Casado G., 1993, ApJ 402, 398 (SSG)

Salvador-Solé E., González-Casado G., Solanes J.M., 1993, ApJ 410, 1

Stein P., 1996, A\&AS 116, 203

Stein P., 1997, A\&A 317, 670

Stein P., Jerjen H., Federspiel M., 1997, A\&A 327, 952

Tadhunter C.N., Morganti R., di Serego Alighieri S., Fosbury R.A.E., Danzinger I.J., 1993, MNRAS 263, 999

Tonry J., Davis M., 1979, AJ 84, 1511

Wall J.V., Peacock J.A., 1985, MNRAS 216, 173

Yahil A., Vidal N.V., 1977, ApJ 214, 347 